\title{
Evaluación del bloqueo del cuadrado lumbar transmuscular ecoguiado en pediatría
}

\author{
Efficacy of ultrasound-guided transmuscular \\ quadratus lumborum block in children
}

Diego S. Guardabassi,2, Carolina Aisenstein², Lara Errecalde², Florencia García Pugliese², Roxana Lettini²

\begin{abstract}
Objectives: Transmuscular quadratus lumborum block (TQLB) is a novel regional anaesthesia technique that has proven to be effective for postoperative pain reduction in different abdominal surgical procedures. The present study evaluated its efficacy on pain intensity and analgesic consumption in children undergoing low abdominal surgery. Methods: The study included forty patients, aged 1 to 6 years, scheduled for low abdominal surgery (hernia repair or orchiopexy) under general anaesthesia. They were enrolled in two groups: TQLB block plus systemic analgesia (group $1 ; n=20$ ) versus wound infiltration done by the surgeon plus systemic analgesia (Group 2; $n=20$ ). All blocks were performed by the same anesthesiologist under general anaesthesia before surgery. Both groups received the same systemic analgesia protocol. Analgesic consumption (ibuprofen) within the first 24 postoperative hours, pain intensity scores (FLACC scale) at 60 minutes, 2, 6 and 24 hours after surgery, time in which the first analgesia was required, satisfaction levels of the parents (0-10), adverse events related to systemic analgesia and time to hospital discharge were evaluated and registered. Results: We found differences between both groups in ibuprofen consumption (80 mg vs $185 \mathrm{mg} ; \mathrm{p}<0.05$ ) and pain scores (FLACC) within the first 24 postoperative hours at each interval $(p<0.05$ for every point in time analyzed). Time in which the first analgesia was required was longer for the TQLB group (18 vs 10 hours; $p<0.05$ ). Satisfaction levels of the parents were also higher in the first group $(p<0.05)$. Adverse events related to medication and time to hospital discharge showed similar results. Further investigation comparing the TQLB with different approaches of QLB or conventional TAP block could be interesting and is required in a near future.
\end{abstract}

\section{Key words:}

Regional anaesthesia, quadratus lumborum block, paediatrics, postoperative analgesia

Servicio de Anestesiología. Hospital del Niño de San Justo. Buenos Aires, Argentina.

Servicio de Anestesiología. Hospital J.M. Ramos Mejía. Ciudad de Buenos Aires, Argentina.

Fecha de recepción: 02 de diciembre de 2019

Fecha de aceptación: 02 de enero de 2020

\section{ORCID}

https://orcid.org/0000-0002-5224-757X

Correspondencia:

Diego S. Guardabassi

Email: diego.guardabassi23@gmail.com 


\section{RESUMEN}

Introducción: El bloqueo del cuadrado lumbar transmuscular (TQL) es una técnica de anestesia regional ecoguiada que demostró ser efectiva en cirugía abdominal abierta y laparoscópica como parte de un esquema multimodal de analgesia postoperatoria. La presente investigación evaluó su eficacia en cirugía de hernia inguinal y testículo no descendido en población pediátrica (de 1-6 años de edad). Materiales y Métodos: El estudio fue diseñado como un ensayo clínico controlado, aleatorizado en 2 ramas: grupo protocolo bloqueo TQL (Grupo 1; $\mathrm{n}=20$ ) vs grupo protocolo infiltración local por cirujano (Grupo $2 ; n=20$ ), simple ciego que incluyó a 40 pacientes pediátricos sometidos a una cirugía de hernia inguinal u orquidopexia unilateral. Todos los bloqueos fueron realizados bajo anestesia general antes del inicio de procedimiento quirúrgico. Ambos grupos recibieron el mismo esquema de analgesia endovenosa y oral postoperatoria. Consumo de analgésicos durante las primeras 24 horas (mg de ibuprofeno), intensidad de dolor con escala FLACC a tiempo 1, 2, 6 y 24 horas postoperatorias, tiempo transcurrido a la primera administración de ibuprofeno, escala de satisfacción parental (0-10) e incidencia de náuseas y vómitos fueron evaluados y registrados. Se consideró como significativa una $p<0,05$. Resultados: El consumo promedio de ibuprofeno en las primeras 24 horas fue de $80 \mathrm{mg}$ para el grupo bloqueo TQL y de $185 \mathrm{mg}$ para el segundo grupo, diferencia estadísticamente significativa $(p<0,05)$. El tiempo transcurrido a la primera administración de ibuprofeno fue mayor en el grupo TQL (18 vs 10 horas), $p<0,05$. Las escalas de dolor por FLACC a los 60 y 120 minutos y a las 6 y 24 horas. postcirugía fueron menores comparadas con el grupo de infiltración local por el cirujano ( $p<0,05$ en cada punto de análisis). Las escalas de satisfacción parental mostraron puntajes más elevados para el grupo que recibió el bloqueo TQL $(p<0,05)$. No se hallaron diferencias significativas en la incidencia de episodios de náuseas y/o vómitos en las primeras 24 horas. postoperatorias $(p>0,2)$. No se reportaron complicaciones asociadas al bloqueo. Conclusiones: Este estudio muestra que el bloqueo TQL aporta beneficios adicionales respecto al plan de analgesia estándar empleado en cirugía de hernia inguinal unilateral u orquidopexia, disminuyendo el requerimiento de analgésicos y la intensidad de dolor en las primeras 24 horas postoperatorias, así como también incrementando la satisfacción de los cuidadores respecto al estado postoperatorio del niño. Sería interesante llevar a cabo nuevas investigaciones comparando esta técnica con otros abordajes del bloqueo de cuadrado lumbar y con el cuestionado bloqueo TAP convencional.
Palabras clave:

Anestesia regional, bloqueo del cuadrado lumbar, pediatría, analgesia postoperatoria

\section{Introducción}

as cirugías de hernia inguinal y testículo no descendido constituyen procedimientos quirúrgicos muy frecuentes en la población pediátrica. Ambos generan un dolor postoperatorio de intensidad variable que requiere un régimen de tratamiento multimodal que provea una eficacia analgésica óptima y minimice los efectos adversos de las drogas utilizadas, teniendo en cuenta que la mayoría se realizan en forma ambulatoria[1]-[5].
El control actual del dolor postoperatorio es pobre. Reportes recientes indican que hasta el $55 \%$ de los niños reportan dolor postoperatorio de intensidad al menos moderada. Y entre 5 al 20\% pueden evolucionar a un dolor crónico que limite su actividad física, intelectual y desarrollo[2]-[3]. Ante esta realidad, el uso de abordajes alternativos en el tratamiento del dolor en este tipo de cirugías es requerido. Surge entonces el empleo de anestesia regional como una alternativa atractiva.

Un componente importante en la fisiopatología 
del dolor postoperatorio en estos procedimientos es el somático, derivado de la inervación de la pared abdominal[6]. El bloqueo del cuadrado lumbar transmuscular (TQL) es una técnica nobel de anestesia regional que consiste en el bloqueo de los nervios sensitivos (T8-L1) que inervan la pared anterolateral del abdomen. Realizada con guía ecográfica para abordar el plano adecuado (entre el músculo cuadrado lumbar y psoas) constituye una técnica segura y de fácil realización, con escasas complicaciones reportadas[7]-[11].

La eficacia de dicho bloqueo en analgesia postoperatoria fue probada en numerosos estudios y distintos tipos de poblaciones y procedimientos[12]-[15]. Sin embargo, su utilidad ha sido escasamente investigada para este tipo de cirugías, en comparación con el esquema estándar (analgesia endovenosa más infiltración local realizada por el cirujano).

Se condujo la presente investigación con el objetivo primario de evaluar si el empleo de un bloqueo TQL guiado con ecografía en pacientes sometidos a cirugía de reparación de hernia inguinal u orquidopexia reduce el requerimiento de analgésicos y las escalas de dolor en las primeras 24 horas del postoperatorio.

\section{Materiales y Métodos}

El presente estudio fue diseñado como un ensayo clínico, controlado, simple ciego, aleatorizado en dos ramas: grupo bloqueo TQL ecoguiado (grupo 1) - grupo infiltración local por cirujano (grupo 2), que incluyó a 40 pacientes pediátricos sometidos a cirugía de hemiabdomen inferior en forma programada.

Se establecieron como criterios de inclusión: pacientes de 1-12 años de edad; ASA I-II, sometidos a cirugía programada de abdomen inferior (hernia inguinal unilateral - testículo no descendido). Y como criterios de exclusión: infección en el sitio de inyección; pacientes con alergia conocida a alguna de las drogas en estudio; coagulopatía; pacientes con patología neurológica/psiquiátrica.

Luego de presentado el protocolo y obtenida la aprobación del Comité de Docencia e Investigación y del Comité de Bioética del Hospital del Niño de San Justo, se dio inicio a la investigación. La misma se llevó a cabo en nuestra institución, entre septiembre de 2018 y mayo de 2019. Se incluyeron a 40 pacientes a los cuales se les realizó una cirugía de abdomen inferior en forma programada.

El proceso de reclutamiento se basó en un muestreo de tipo no probabilístico por serie de casos consecutivos. Los pacientes candidatos de ser incluidos, una vez firmado el consentimiento informado por parte de sus padres, fueron asignados aleatoriamente a alguna de las dos ramas de tratamiento antes de la cirugía, mediante el método de sobres cerrados.

\section{Se estableció el siguiente protocolo:}

Preoperatoriamente todos los pacientes fueron premedicados con midazolam vía oral $(0,5 \mathrm{mg} / \mathrm{kg})$ o endovenoso $(0,04 \mathrm{mg} / \mathrm{kg})$ en caso de tener acceso venoso previo.

Una vez en el quirófano, se le colocaron los monitores estándar: oxímetro de pulso, electrocardiografía y presión arterial no invasiva. Si el paciente presentaba acceso venoso, la anestesia general se indujo con propofol $3 \mathrm{mg} / \mathrm{kg}$, previa administración de lidocaína $0,5 \mathrm{mg} / \mathrm{kg}$ endovenosa para disminuir dolor a la inyección. Si el paciente no poseía acceso venoso, la inducción fue inhalatoria con máscara facial y sevofluorano. Una vez alcanzado el plano adecuado, se procedió a la canalización de un acceso venoso. Todos los pacientes recibieron fentanilo 2 ug/kg endovenoso y se les colocó una máscara laríngea (IntaventOrthofix, Maidenhead, United Kingdom) de tamaño apropiado para asegurar la vía aérea. El mantenimiento anestésico se realizó con sevofluorano y $\mathrm{O}_{2}$ al $50 \%$. Dosis adicionales de fentanilo ( $1 \mathrm{ug} / \mathrm{kg}$ endovenoso) fueron administradas a criterio del anestesiólogo a cargo en base a la respuesta al estímulo quirúrgico (aumento de más del 20\% del basal de la frecuencia cardíaca y/o tensión arterial) o cambios en el patrón ventilatorio.

En los pacientes aleatorizados a recibir el bloqueo TQL, luego de la inducción a anestesia general y colocación de máscara laríngea, se realizó el mismo guiado por ecografía (ecógrafo Sonosite M-Turbo; Sonosite Inc, Bothell, WA 98021, USA) con transductor lineal de entre 6 a $13 \mathrm{Mhz}$, en forma unilateral del lado a intervenir. Se colocó al paciente en decúbito lateral opuesto al lado correspondiente y se efectuó antisepsia de la zona con iodopovidona. Se colocó la sonda ecográfica lineal entre la cresta ilíaca y el reborde costal inferior, con una cubierta estéril. El músculo oblicuo externo fue escaneado postero lateralmente hasta su borde posterior, hasta identificar los músculos cuadrado lumbar y psoas. Una vez halladas y visualizadas las estructuras adyacentes (apófisis transversa-músculo erector espinal-fascia transversalis), se procedió a realizar la punción utilizando una aguja SonoTAP de 22G y 50 mm (Pajunk, Geisingen, Alemania) insertada con un abordaje ecográfico en plano. Al alcanzar la punta de la aguja el plano entre el cuadrado lumbar y psoas y luego de obtener una aspiración negativa para sangre, se inyectaron $0,5 \mathrm{~mL} / \mathrm{kg}$ de bupivacaìna 0,25\% (máximo $20 \mathrm{~mL}$ ). El bloqueo 
fue realizado por un anestesiólogo con experiencia en anestesia regional, que ha realizado al menos 30 bloqueos previamente. La solución anestésica local empleada fue preparada por el mismo anestesiólogo.

En los pacientes aleatorizados a recibir infiltración local realizada por el cirujano a cargo, luego de la inducción a anestesia general y colocación de máscara laríngea, se realizó el bloqueo infiltrativo. Tomando como reparo la espina ilíaca anterosuperior, el punto de inyección fue establecido $2 \mathrm{~cm}$ por dentro y $2 \mathrm{~cm}$ por arriba de su punto más saliente. Con una aguja de inyección intramuscular, la punción se realizó en forma perpendicular a todos los planos, atravesando dos resaltos al ingresar con la misma en profundidad (fascias musculares). Una vez experimentado el segundo resalto, previa aspiración negativa de sangre, se procedió a inyectar $0,5 \mathrm{~mL} / \mathrm{kg}$ de bupivacaína 0,25\% (máximo $20 \mathrm{~mL}$ ).

En ambos grupos se administró: dexametasona $0,2 \mathrm{mg} / \mathrm{kg}$ ev. y dipirona $20 \mathrm{mg} / \mathrm{kg}$ ev. al inicio del procedimiento en bolo lento.

El dolor postoperatorio fue evaluado mediante escala FLACC en todos los casos. Cuando el score de FLACC en la unidad de cuidados postanestésicos fue de 4 o mayor, se administró fentanilo $1 \mathrm{ug} / \mathrm{kg}$ endovenoso lento. En el piso de internación, $10 \mathrm{mg} / \mathrm{kg}$ de ibuprofeno oral fueron administrados si el paciente presentaba un score de FLACC mayor a 2. Los padres/ tutores fueron informados e instruidos en la evaluación del dolor, y si los pacientes experimentaban dolor en su hogar, les administraron $10 \mathrm{mg} / \mathrm{kg}$ de ibuprofeno oral, registrando tal evento en una planilla con horario. Todos los datos necesarios postalta hospitalaria, fueron recogidos por el anestesiólogo por vía telefónica.

En cuanto a las variables de resultado analizadas, se evaluó como resultado primario la cantidad de analgésicos consumidos durante las primeras 24 horas del postoperatorio (mg de ibuprofeno).

Como resultados secundarios se evaluaron:

1. Escala de dolor durante las primeras 24 horas con escala FLACC, en rango de 0-10 según Tabla 1, a tiempo 1, 2, 6 y 24 horas postoperatorias. Tiempo 0 : definido como hora de salida del paciente de quirófano.

2. Tiempo transcurrido a la primera administración de ibuprofeno en las primeras 24 horas.

3. Escala de satisfacción parental (numérica de 0-10) a tiempo 6 y 24 horas postoperatorias. $(0=$ nada/ poco conforme).

4. Incidencia de náuseas (sí/no) y vómitos (números de episodios) a tiempo 1, 2, 6 y 24 horas postoperatorias.
Todos los datos fueron volcados en una planilla de evaluación diseñada a tal fin. Los pacientes fueron codificados según el número del sobre de aleatorización.

\section{Cálculo del tamaño muestral:}

Se efectuó el cálculo del tamaño muestral, a partir de la variable consumo de ibuprofeno en las primeras 24 horas. del postoperatorio en miligramos (mg), con los datos obtenidos de estudios previos similares. Se estableció, además, para el cálculo, un nivel de confianza prefijado para una seguridad del $95 \%$ y un poder del $80 \%$, considerándose también una eventual pérdida del $10 \%$ de los pacientes incluidos en el estudio.

Análisis estadístico:

Fue realizado utilizando un programa estándar (STATA). La normalidad de los datos fue analizada mediante el test de Kolmogorov-Smirnov. Las variables cuantitativas continuas se analizaron mediante $t$ de Student o Mann Whitney acorde a distribución. Las variables categóricas fueron analizadas mediante test de chi cuadrado o test exacto de Fisher. Se evaluó el cambio del dolor postoperatorio por escala FLACC con un modelo lineal generalizado para medidas repetidas en los tiempos 1, 2, 6 horas y 24 horas. Se ajustó por potenciales confundidores con un modelo de lineal generalizado múltiple. Se informan los intervalos de confianza calculados para cada caso. Los datos normalmente distribuidos son presentados como media +- desvío estándar, los no-normalmente distribuidos como mediana (rango) y los categóricos como data cruda y frecuencias.

\section{Resultados}

El consumo promedio de ibuprofeno en las primeras 24 horas fue de $80 \mathrm{mg}$ para el grupo bloqueo TQL y de $185 \mathrm{mg}$ para el segundo grupo, diferencia estadísticamente significativa $(p<0,05)$. El tiempo transcurrido a la primera administración de ibuprofeno fue mayor en el grupo TQL (18 vs 10 horas), $p<$ 0,05 . Las escalas de dolor por FLACC a los 60 y 120 minutos y a las 6 y 24 horas. postcirugía fueron menores comparadas con el grupo de infiltración local por el cirujano ( $p<0,05$ en cada punto de análisis). Las escalas de satisfacción parental mostraron puntajes más elevados para el grupo que recibió el bloqueo TQL ( $p$ $<0,05)$. No se hallaron diferencias significativas en la incidencia de episodios de náuseas y/o vómitos en las primeras 24 horas. postoperatorias $(p>0,2)$. No se reportaron complicaciones asociadas al bloqueo. 


\begin{tabular}{|c|c|c|c|}
\hline Características & Grupo 1 & Grupo 2 & $\mathbf{P}$ \\
\hline Mediana de edad, años (rango) & $2(1-6)$ & $3(1-6)$ & 0,52 \\
\hline Consumo de ibuprofeno 24 horas, mg (DE) & $80(12,31)$ & $185(28,75)$ & 0,002 \\
\hline FLACC dolor 60 minutos, media (DE) & $0,44(0,58)$ & $0,96(0,73)$ & 0,01 \\
\hline FLACC dolor 120 minutos & $0,12(0,33)$ & $0,64(0,70)$ & 0,02 \\
\hline FLACC dolor 6 horas & $0(0)$ & $0,36(0,49)$ & 0,001 \\
\hline FLACC dolor 24 horas & $0,04(0,20)$ & $0,28(0,45)$ & 0,022 \\
\hline Escala de satisfacción parental, media (DE) & $9(0,5)$ & $8,32(0,74)$ & 0,001 \\
\hline Tiempo al alta, horas (rango) & $6(5-7)$ & $6(5-8)$ & 0,77 \\
\hline Náuseas y vómitos (n) & $5 \%(2)$ & $10 \%(4)$ & 0,26 \\
\hline
\end{tabular}

\section{Discusión}

En la presente investigación hallamos que el empleo del bloqueo TQL ecoguiado, sumado al plan de analgesia multimodal postoperatoria estándar en cirugía de abdomen inferior en pediatría, contribuye a disminuir el consumo de analgésicos y la intensidad de dolor medida por escala FLACC en las primeras 24 horas postoperatorias, incrementando, además, las escalas de satisfacción parental. Este hallazgo no resultó llamativo para los autores, dado que infiltración con anestésico local es una técnica estándar realizada por el cirujano a ciegas y cuyo target (espacio entre la fascia del oblicuo menor y transverso) en casi un $50 \%$ de los casos no es alcanzado en forma precisa. Sumado a esto, las potenciales complicaciones hacen que este abordaje no sea seguro para los pacientes. La presente investigación demuestra la superioridad de una técnica óptima, apropiada y segura y brinda un marco para incrementar la calidad y seguridad en la atención del paciente pediátrico.

El bloqueo del cuadrado lumbar ha sido estudiado en numerosas publicaciones, contribuyendo, como parte de un plan analgésico multimodal, al manejo del dolor postoperatorio en cirugía abdominal abierta y laparoscópica, en cuanto a reducción de la intensidad del dolor, consumo de opioides y efectos adversos. Sin embargo, éstas resultan bastante heterogéneas en cuanto a las poblaciones analizadas y técnicas empleadas, por lo que la comparación con nuestra investigación resulta dificultosa. Respecto a la técnica realizada, algunas publicaciones no describían la técnica en forma precisa, ni qué experiencia en la misma tenía el profesional que la realizaba. Considero a éste un punto fuerte en nuestro estudio, donde todos los bloqueos fueron realizados por el mismo anestesió- logo con experiencia en anestesia regional (al menos 50 bloqueos realizados previamente) y la técnica fue descripta pertinentemente. Cabe aclarar también que la comparación con otras técnicas de bloqueo de la pared abdominal (ilioinguinal, iliohipogástrico, vaina de los rectos) u otros tipos de abordaje del bloqueo TAP (posterior, cuadrado lumbar) nunca fue considerado en el diseño ni representó uno de los objetivos de nuestra investigación.

En cuanto a la población estudiada, este tipo de cirugías se realizan en pacientes vulnerables, cuya patología supone, además de lo físico, una carga psíquica y emocional importante y que requieren, en nuestro medio, del empleo de un plan de analgesia multimodal que incluya un esquema óptimo para el control del dolor postoperatorio. Estas características le confieren a este grupo una identidad propia, por lo que comparar con otras poblaciones bastante disímiles no resulta pertinente.

Consideramos que nuestra investigación, desde lo metodológico, fue objetivamente correcta, con un tamaño muestral adecuado y representativo. Todas las evaluaciones fueron realizadas por un observador independiente, eliminando la posibilidad de sesgos a este nivel.

Existen, sin embargo, una serie de limitaciones en nuestro estudio. En primer lugar, no haber considerado el análisis en la muestra de los factores de riesgo de náuseas y vómitos postoperatorios ni de aquellos asociados a mayor dolor postoperatorio (por ejemplo, técnica quirúrgica y tiempo de cirugía). La segunda limitación fue representada por la ausencia de doble ciego, ya que el grupo control no recibió un bloqueo con placebo. Cuestiones éticas fueron determinantes en nuestra elección.

Por último, en la mayoría de los centros hospita- 
larios este tipo de cirugías se realiza en forma ambulatoria, con un régimen de analgesia multimodal vía oral para el control del dolor postoperatorio. En nuestro caso, todos los pacientes fueron dados de alta el mismo día de la cirugía. Esto nos permitió evaluar de forma precisa y comparativa el consumo de analgésicos entre ambos grupos. Creemos que esto constituye una fortaleza importante para comparar nuestro trabajo con otros, realizados en distintos contextos.

Concluimos, entonces, que los resultados de este estudio indican que el bloqueo TQL aporta un beneficio en términos de duración y efectividad analgésica respecto a la infiltración local realizada por el cirujano en cirugía de abdomen inferior.

No consideramos, sin embargo, pertinente excluir la posibilidad que alguna investigación, con un diseño diferente, pueda mostrar distintos resultados en términos de consumo de analgésicos y dolor postoperatorio, por lo que sería interesante sea objeto de nuevas líneas de trabajo. Otro punto interesante a futuro sería investigar la eficacia de este abordaje en forma comparativa con otros bloqueos previamente descriptos (bloqueo del cuadrado lumbar 1 y 2, bloqueo TAP, bloqueo ilioinguinal-iliohipogástrico).

\section{Referencias}

1. Guideline statement: management of procedure-related pain in children and adolescents. J Paediatr Child Health. 2006 Feb;42(S1 Suppl 1):S1-29. https://doi.org/10.1111/j.14401754.2006.00798_1.x PMID:16626312

2. Perrott C, Lee CA, Griffiths $S$, Sury MR. Perioperative experiences of anesthesia reported by children and parents. Paediatr Anaesth. 2018 Feb;28(2):14956. https://doi.org/10.1111/ pan.13300 PMID:29266767

3. Young KD. Pediatric procedural pain. Ann Emerg Med. 2005 Feb;45(2):160-71. https://doi.org/10.1016/j. annemergmed.2004.09.019 PMID:15671974

4. Good Practice in Postoperative and Procedural Pain. 2nd ed. 2012.

5. Royal College of Nursing (UK). Clinical Guidelines for the Recognition and Assessment of Acute Pain in Children. London Royal College of Nursing; 2009.

6. Netter FH Back and Spinal Cord (Netter FH, editor). Atlas of Human Anatomy Summit. New Jersey, USA: The Ciba-Geigy Corporation; 1989. pp. 145-55.

7. Murouchi T. Quadratus lum- borum block intramuscular approach for pediatric surgery. Acta Anaesthesiol Taiwan. 2016 Dec;54(4):135-6. https://doi. org/10.1016/j.aat.2016.10.003 PMID:27939905

8. Visoiu M, Yakovleva N. Continuous postoperative analgesia via quadratus lumborum block - an alternative to transversus abdominis plane block. Paediatr Anaesth. 2013 Oct;23(10):95961. https://doi.org/10.1111/ pan.12240 PMID:23927552

9. Carline L, McLeod GA, Lamb C. A cadaver study comparing spread of dye and nerve involvement after three different quadratus lumborum blocks. Br J Anaesth. 2016 Sep;117(3):38794. https://doi.org/10.1093/bja/ aew224 PMID:27543534

10. Børglum J, Gögenür I, Bendtsen TF. Abdominal wall blocks in adults. Curr Opin Anaesthesiol. 2016 Oct;29(5):638-43. https://doi.org/10.1097/ AC0.0000000000000378 PMID:27429253

11. Murouchi T, Iwasaki S, Yamakage M. Quadratus lumborum block: analgesic effects and chronological ropivacaine concentrations after laparoscopic surgery. Reg Anesth Pain Med. 2016 Mar-Apr;41(2):146-50. https://doi.org/10.1097/
AAP.0000000000000349 PMID:26735154

12. Chakraborty A, Goswami J, Patro V. Ultrasound-guided continuous quadratus lumborum block for postoperative analgesia in a pediatric patient. A A Case Rep. 2015 Feb;4(3):346. https://doi.org/10.1213/ XAA.0000000000000090 PMID:25642956

13. Baidya DK, Maitra S, Arora MK, Agarwal A. Quadratus lumborum block: an effective method of perioperative analgesia in children undergoing pyeloplasty. J Clin Anesth. 2015 Dec;27(8):694-6. https:// doi.org/10.1016/j.jclinane.2015.05.006 PMID:26174113

14. Blanco R, Ansari T, Girgis E. Quadratus lumborum block for postoperative pain after caesarean section: A randomised controlled trial. Eur J Anaesthesiol. 2015 Nov;32(11):8128. https://doi.org/10.1097/ EJA.0000000000000299 PMID:26225500

15. Blanco R, Ansari T, Riad W, Shetty N. Quadratus lumborum block versus transversus abdominis plane block for postoperative pain after cesarean delivery: a randomized controlled trial. Reg Anesth Pain Med. 2016 Nov/Dec;41(6):757- 
62. https://doi.org/10.1097/ AAP.0000000000000495 PMID:27755488

16. Manworren RC, Hynan LS Clinical validation of FLACC: preverbal patient pain scale. Pediatr
Nurs. 2003 Mar-Apr;29(2):140-6. PMID:12723828

17. Hicks $C L$, von Baeyer $C L$, Spafford PA, van Korlaar I,

Goodenough B. The Faces

Pain Scale-Revised: toward a common metric in pediatric pain measurement. Pain. 2001 Aug;93(2):173-83.

https://doi.org/10.1016/

S0304-3959(01)00314-1

PMID:11427329 